\title{
BIBLOS for simulation of the Earth observation missions
}

Robert Kędzierawski, Wojciech Oryszczak, Karolina Szczepankiewicz, Adriano Camps, Hyuk Park, et al.

Robert Kędzierawski, Wojciech Oryszczak, Karolina Szczepankiewicz, Adriano Camps, Hyuk Park, Pedro A. Barrios Garcia, "BIBLOS for simulation of the Earth observation missions," Proc. SPIE 11442, Radioelectronic Systems Conference 2019, 1144204 (11 February 2020); doi: $10.1117 / 12.2565755$

SPIE. Event: Radioelectronic Systems Conference 2019, 2019, Jachranka, Poland 


\title{
BIBLOS for simulation of the Earth Observation missions
}

\author{
Robert Kedzierawski ${ }^{a}$, Wojciech Oryszczak ${ }^{a}$, Karolina Szczepankiewicz ${ }^{\mathrm{a}}$, Adriano Camps ${ }^{\mathrm{b}}$, \\ Hyuk Park ${ }^{b}$, and Pedro A. Barrios Garcia ${ }^{c}$ \\ ${ }^{a}$ GMV Innovating Solutions Sp z o.o., ul. Hrubieszowska 2, 01-209 Warsaw, Poland \\ ${ }^{\mathrm{b}}$ CommSensLab-UPC, Universitat Politecnica de Catalunya (UPC) and Institut d'Estudis \\ Espacials de Catalunya IEEC/CTE-UPC, c/Jordi Girona, 1-3 Barcelona, Spain \\ 'ESA/ESTEC, Keplerlaan 1, 2201 AZ Noordwijk, The Netherlands
}

\begin{abstract}
End-to-End Mission Performance Simulators for Earth Observation missions are one of the prominent tools for system design and scientific validation in early mission phases. The European Space Agency has promoted efforts to exploit synergies between activities and reduce engineering costs in mission planning. The main goal of the BIBLOS project is to provide a library of software units called "Building Blocks", or simply "Blocks". These blocks could then be integrated into modules, following the reference architecture developed in the frame of the ARCHEO-E2E project, in order to build an End-to-End Simulator in an efficient manner. The first stage of BIBLOS project, referred to as EOMODEL (or BIBLOS-1) and, focused on Passive Optical instruments, was successfully finished (in April 2016) with the geometry, scene generation and instrument modelling of optical imagers, which are one of the most frequent types of passive optical instrument on Earth Observation satellites.

BIBLOS-2, which is the second stage of the BIBLOS project, is a currently finished activity with the purpose of expanding the BIBLOS library to include Passive Microwaves instruments and Active Microwaves instruments. Additionally, as part of a continuous improvement process, this second stage of BIBLOS has also updated some of the most computational performance intensive blocks for Passive Optical instruments with parallel implementation for Graphic Processing Units. For BIBLOS-2, the Sentinel-1 and MicroWave Imager (MWI) instruments have been selected to develop the building blocks for Active Microwaves and Passive Microwaves instruments respectively.

During the BIBLOS-2 stage the full simulation chain has been developed for Passive Microwave, starting from the Geometry Module, through Scene Generation and Instrument Modules and ending at Level 1 Processing Module. For Active Microwave Instruments this stage of the BIBLOS activity is focused on the Geometry Module and the Performance Evaluation Module only. Special efforts are devoted to adapt the Geometry Module for monostatic, bistatic and multistatic missions in various configurations and with different a number of microwave instruments. In addition, the Performance Evaluation Module is in charge of evaluating the performance figures and providing plots, such as diamond diagram, ambiguity area and ratios, Noise Equivalent Sigma Zero and antenna radiation patterns.
\end{abstract}

This paper presents the work successfully carried out during the BIBLOS-2 stage, currently performed by GMV under ESA contract.

Keywords: Earth Observation, mission performance simulation, microwave systems, optical systems, synthetic aperture radar, conical scanning microwave radiometer

Further author information: (Send correspondence to BIBLOS Team, Pedro A. Barrios Garcia)

BIBLOS Team: E-mail: biblos@gmv.com, Telephone: +48 223955165

Pedro A. Barrios Garcia: E-mail: pedro.barrios@esa.int, Telephone: +31 715654782

Radioelectronic Systems Conference 2019, edited by Piotr Kaniewski, Jan Matuszewski, Proc. of SPIE Vol. 11442, $1144204 \cdot$ (c) 2020 SPIE · CCC code: 0277-786X/20/\$21 · doi: 10.1117/12.2565755 


\section{INTRODUCTION}

The BIBLOS [1] project is designed to guide and help you with the development of an End-to-End (E2E) Mission Performance Simulators (E2ES) for Earth Observation (EO) missions. An E2ES is a tool used by the scientists and engineering teams to simulate the acquisition and generation of the satellite products. There are an increasing number of missions that develop performance simulators in early stages (phase A, B1) to assess requirements, evaluate the instrument characteristics, the products, etc. These simulators often evolve into the operational simulators. The development of a simulators is costly. There needs to be a detailed definition of the architecture for each mission, implementation and a lengthy validation process. To try to reduce the cost, and avoid re-engineering, the BIBLOS project has a step-by-step approach that:

- Helps you define an architecture taking into account your mission particularities

- Provides validated software units that are ready to be used

The paper is organized as follows. Section 2 presents the background and the scope of BIBLOS-2 project. Current version of BIBLOS Software is described in Section 3. Section 4 presents current content of project website significantly updated during the current project phase. Example applications of software components developed in current phase are shown in Section 5. Results shown in this section are generated for passive and active microwave instruments. Passive optical instrument is not considered in this paper. Section 6 concludes paper and shows plans for the Maintenance Period of BIBLOS-2.

\section{BIBLOS-2 BACKGROUND}

BIBLOS-2 project is a continuation of EOMODEL (BIBLOS-1) [2]. All BIBLOS [1] projects are based on and inherit from ESA's ARCHEO-E2E [3] project led by GMV. ARCHEO-E2E has analysed past, on-going and future missions to create the baseline design for Instrument Data Simulator (IDS) and Ground Processor Prototype (GPP) processing chain, called the reference architecture. BIBLOS-2 follows the reference architecture defined and implements it, which means that a software library for creating the IDS and GPP for various ESA missions is the main goal and output artefact of the project. Figure 1 shows generic data flow at the highest level of the reference architecture. Two versions of the reference architecture are presented in [4] and [5]. Furthermore, each Module is composed of several Building Blocks (Block 1, Block 2, etc.), which are the components of the full End-to-End Simulator (E2ES) Chain (Figure 2). As show in Figure 1, there are six High-Level elements in the Reference Architecture. These elements are called Modules, and are defined as follow:

- Geometry Module (GM): In charge of simulating the SC orbit and attitude, as well as the generation of the observation geometry of each instrument.

- Scene Generator Module (SGM): In charge of simulating the scene to be observed (terrain, ocean or atmosphere) and all environmental effects (radiative transfer models, atmosphere simulation, illumination conditions...) to be considered for the correct generation of the stimuli to be entered to the instrument model.

- Instrument Module (IM): In charge of simulating the sensor behaviour, having different outputs depending on the type of instrument.

- Level-1 Processing Module (L1PM): In charge of the generation of level-1 products, from level-1A to level$1 C$.

- Level-2 Retrieval Module (L2RM): In charge of performing the retrieval of the geophysical parameters that are objective of the mission/instrument. Depending on the mission and on its definition of the products, this module would generate level-2 data or products at higher level of processing.

- Performance Evaluation Module (PEM): In charge of performing the needed analysis of the simulator outputs to evaluate the performance of the mission. It could be run at different points of the simulation chain. 


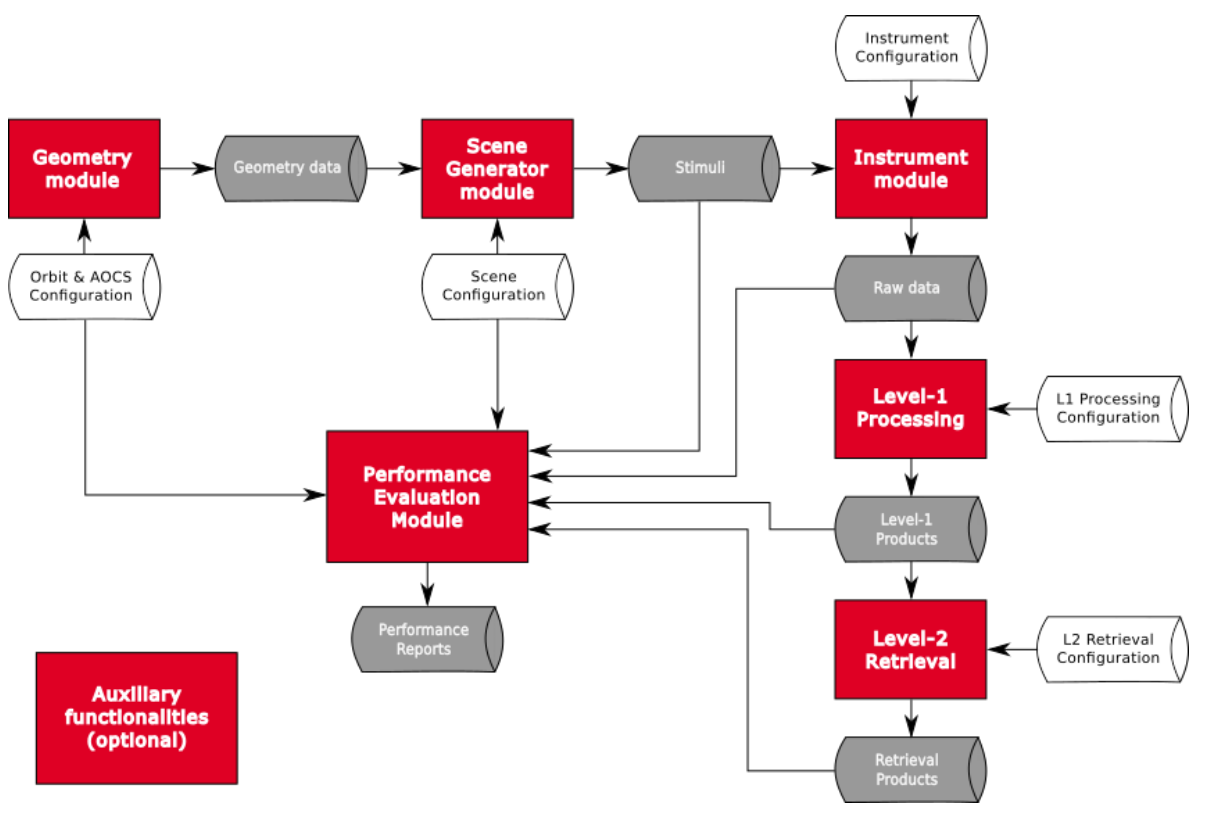

Figure 1. Generic data flow at the highest level of the reference architecture (based on [3]).

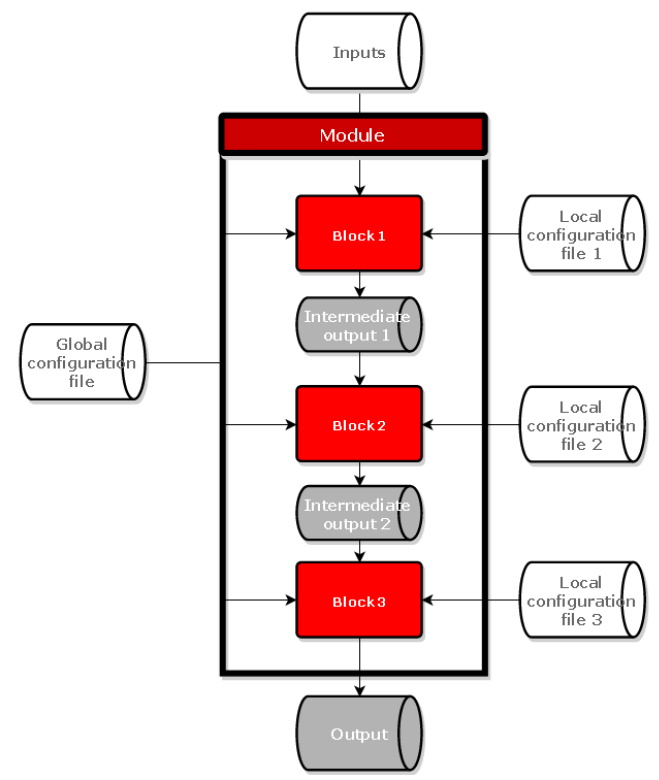

Figure 2. Building Blocks and Module of the Reference Modular Architecture of E2E Mission Performance Simulator (based on [3]).

\section{BIBLOS SOFTWARE}

Table 1 shows current status of BIBLOS software, i.e. Modules (sets of Building Blocks) implemented over various instrument types. More details can be found in documentation shared in [1]. Moreover, new modules planned to be designed, implemented and verified in next phase of BIBLOS are depicted, e.g. BIBLOS-3.

Figure 3 shows currently available Building Blocks within the BIBLOS v2.0 software and required libraries and CFIs. Within the BIBLOS projects the 31 Building Blocks were designed, implemented and verified. In particular, 8 BBs for passive optical, 14 BBs for passive microwave and 9 BBs for active microwave instruments. 
Table 1. Modules (Building Blocks) implemented for various instrument types.

\begin{tabular}{|c|c|c|c|c|}
\hline Module vs. Instruments & Passive Optical & Active Optical & Passive Microwaves & Active Microwaves \\
\hline GM & BIBLOS v2.0 & - & BIBLOS v2.0 & BIBLOS v2.0 \\
\hline SGM & BIBLOS v2.0 & - & BIBLOS v2.0 & BIBLOS v2.0 \\
\hline IM & BIBLOS v2.0 & - & BIBLOS v2.0 & BIBLOS-3 \\
\hline L1PM & - & - & BIBLOS v2.0 & BIBLOS-3 \\
\hline L2RM & - & - & - & - \\
\hline PEM & BIBLOS-3 & - & BIBLOS-3 & BIBLOS v2.0 \\
\hline
\end{tabular}

Detailed description of the algorithms and interfaces is enclosed in documentation provided in [1], in the D3, D4 and D5 documents.

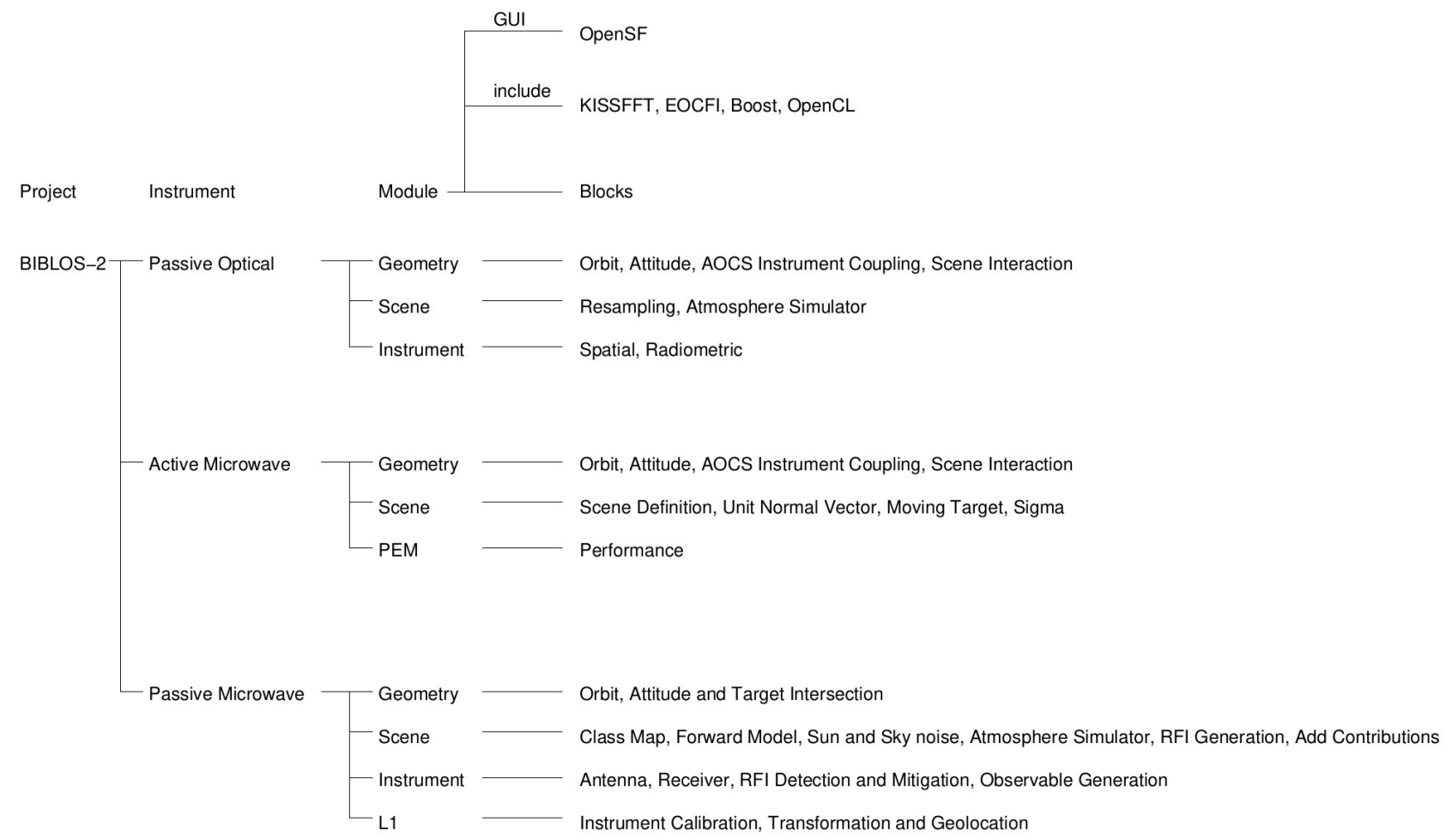

Figure 3. BIBLOS Building Blocks (i.e. components) and required CFI

At least two new versions of BIBLOS software are planned to be released during the Maintenance Period, one Adaptive Release and one Corrective Release, tentatively identified as, respectively, BIBLOS v2.1 (March 2020) and BIBLOS v2.2 (October 2020).

\subsection{Architecture}

Software architecture and design compose reference architecture (Figure 1). The Instrument is the main design driver for a simulator. The Module is a stage of the simulation chain. The simulation chain of any Earth Observation end-to-end simulator can be divided into 6 basic Modules (Figure 1). Each Module is composed 
by Blocks. A Block is a unit of software that performs one functionality, for example the Orbit Block, the Attitude Block, the Line-of Sight Block, etc. The Blocks are the "bricks" of the simulation chain.

BIBLOS components are implemented in $\mathrm{C}++$ language and support reading and writing of NetCDF and XML files. Within several components the functionalities provided by EOCFI [6] are used to provide orbit propagation and target pointing. All BIBLOS components (BBs depicted in Figure 3) have been developed in

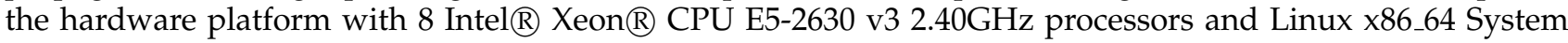
with kernel 4.6.4-5.

\subsection{Software Validation}

Building Blocks (the bricks of E2ES) have been validated and verified at various level. Detailed plans for software verification and verification are described in documents D6 and D7 while results are provided in D9 and D10, respectively. Dedicated Proof-of-Concept scenarios are defined to prove BIBLOS usage.

\subsection{Computational Performance}

During the second stage of BIBLOS project several blocks have been analysed to improve computational performance. Parallel computing methods, such as Graphic Processing Units (GPU), Multi-core CPUs, OpenCL and OpenMP programming have been analysed, and as agreed with ESA, OpenCL has been selected for parallelization of software components. With portability, which is main advantage of OpenCL, BIBLOS components can be used and executed also on MAC computers as well as on GNU Operating Systems. Execution time of particular block is highly dependent from block type and amount of data to be processed.

\subsection{Licence}

BIBLOS is developed and released under the European Space Agency Software Community Licence Permissive - v2.3. [7].

\section{BIBLOS WEBSITE}

During the second phase of BIBLOS the project website [1] has been modified, both at layout level and in accessible content. Current layout is shown in Figure 4. Dedicated tabs and menu were created to collect all information about software releases with license terms and conditions, links to external libraries and Software Problem Repots summary. BIBLOS v2.0 software is available for registered and verified users together with Software User Manual, Software Reuse File and Software Configuration File. 'News \& Releases' section is added to provide an information about latest news and software releases.

To facilitate installation procedure the two dedicated automated installation packages are prepared, respectively, for GNU / Linux Ubuntu 16.04 and MacOs 10.12, both around 3.2 GB. Downloaded package can be simply extracted to user-specified location. Script instal.sh is provided to facilitate installation procedure. This script extracts BIBLOS sources and COTS, compiles software, runs verification tests and, finally, compares the generated output with reference data included in the installation package. Large size of installation package results from inclusion of input for instrument test and reference outputs as well as input data and configuration files for Proof of Concept (PoC) scenarios and precompiled COTS. Raw BIBLOS source (30 MB) and not compiled COTS (114 MB) are also provided for registered users. Additional tabs were created for tutorials and Frequently Asked Questions (FAQ). Four tutorials have been released before the end of the 2019, one for Quick Start Guide installation of BIBLOS source, three others with description of Proof-of-Concept scenarios for passive optical and passive microwave instruments and, due to project rescope, with description of Performance Evaluation Module usage for evaluation of bistatic missions of active microwave instruments, for instance, Synthetic Aperture radar (SAR). According to second phase specification and contract, the project website is planned to be updated during the maintenance period of BIBLOS-2.

\subsection{Registration}

BIBLOS v2.0 software and all relevant documentation is available for registered and verified users from academia, research institutions, proved companies, based on licence conditions [7], only from the ESA State Members (22 Countries). All detail can be found at https://gmv-biblos.gmv.com/register/ 


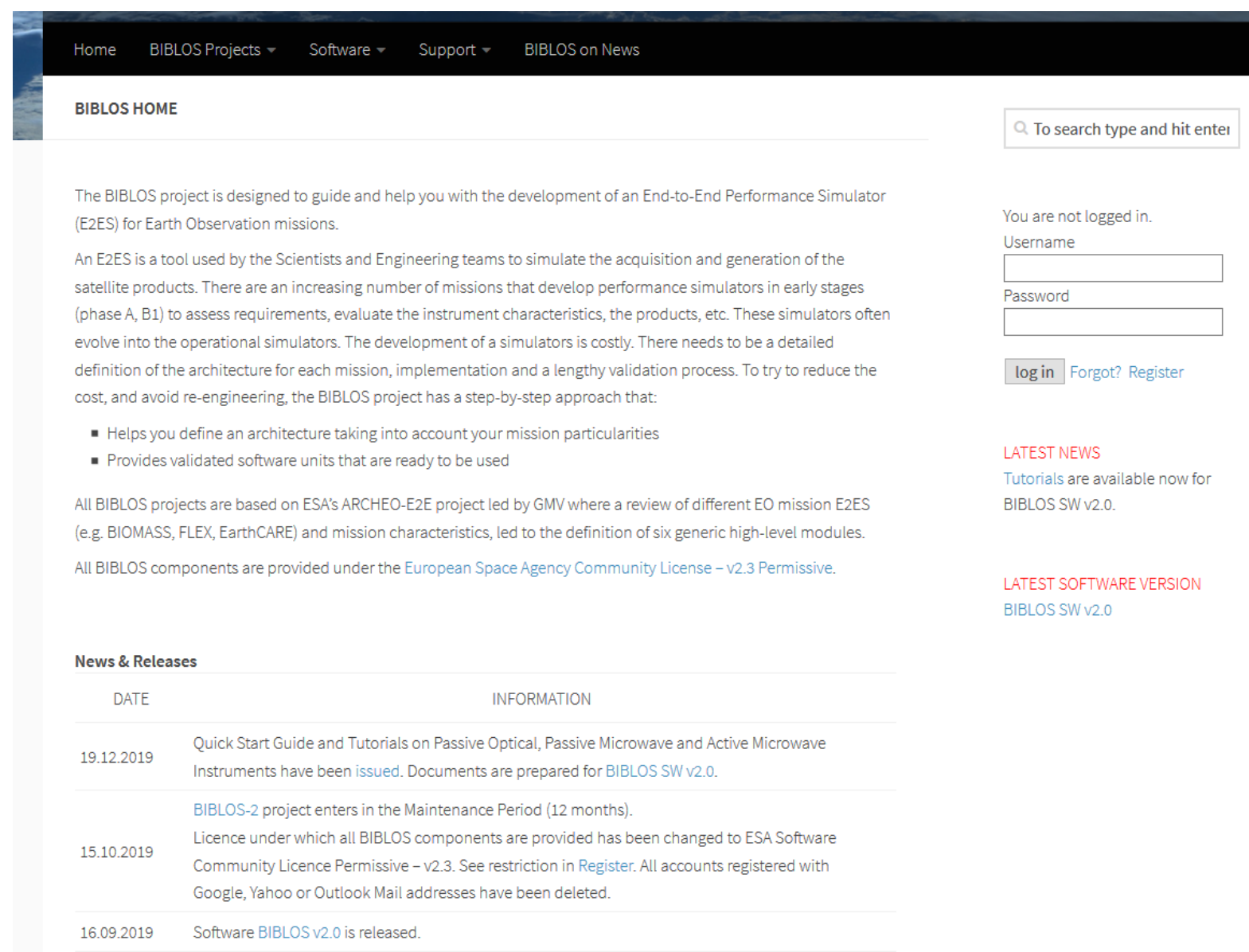

Figure 4. Screenshot of BIBLOS website updated layout https ://gmv-biblos.gmv . com/

\subsection{Project Documentation}

Various documents are available at [1]. Most of them are the project documents prepared in within the BIBLOS2 project (in 'Project Documents' menu of BIBLOS Project/BIBLOS-2 tab). Software requirement specification, BBs detailed design and interfaces description are provided, respectively, in documents D3, D4 and D5. Test plans for verification and validation in D6 and D7. Results of these tests in D9 and D10. Reused files are described in document D12 and user manual in D13. Software release documents are D14 and D15. Proof of concept scenarios for instrument types are defined in D1, with results described in D11 and D21.

\section{E2ES WITH BIBLOS SOFTWARE}

Building Blocks developed or updated during the second stage for BIBLOS activity can be used to simulate wide range of parameters or aspects for Earth observation missions. Example figures shown below are plotted based on the outputs of Building Blocks, as for passive microwave instrument, or directly by the Performance Evaluation Module, as for active microwave instrument.

\subsection{Generic}

Depending on the instrument design each of the modules shown in Figure 2 are composed of various blocks with specific functionalities. Characteristics of observed or illuminated Scene (used in SGM) can be defined differently between instruments depending instrument type. Similarly, raw signal synthesis and on-board processing are determined by instrument type (simulated by IM) as well as signal processing algorithm modelled 
in L1PM. Despite these differences depending on the type of instrument the propagation and attitude calculation is similar for instruments and based on EOCFI [6]. In GM, which is the first module of E2ES, initial orbit setting, pointing configuration and steering parameters are used to initialize a generic satellite or specific ones, as Sentinel-1, MetOp-SG, ENVISAT, CryoSat. Figure 5 shows acquisition geometry (generated Orbit and Attitude files complaint with [6] and [8]) for an example of Synthetic Aperture Radar (SAR) working in Stripmap mode with 4 elevation beams (ScanSAR) and the cross-track conical scanning microwave radiometer. Full orbit (satellite sub-rack position plotted over the geodetic coordinates) is simulated with acquisition of 20 minutes for both instruments. Cross-track scanning is performed with rotation speed of 6.45 rotation per minute (rpm) in 49.5 degrees. Elevation beams for SAR system are defined between 25-30, 29-34, 33-38 and 37-42 degrees. Along-track gaps are observable within observed swath. Figure 6 shows acquisition geometry of conical scanning microwave radiometer along full orbit of 100 minutes.

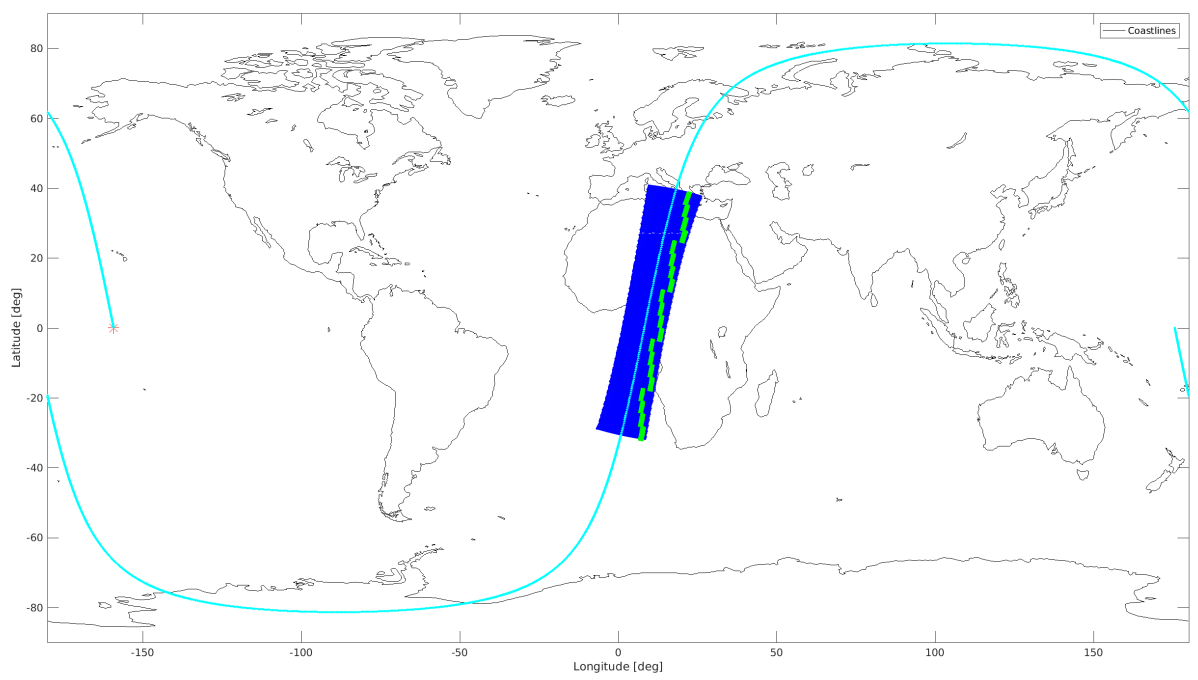

Figure 5. Comparison of pointing and steering for two simulated instrument: SAR (Stripmap mode for 4 beams without TOPS, green areas) and Cross-Track Scanning Microwave Radiometer (blue areas).

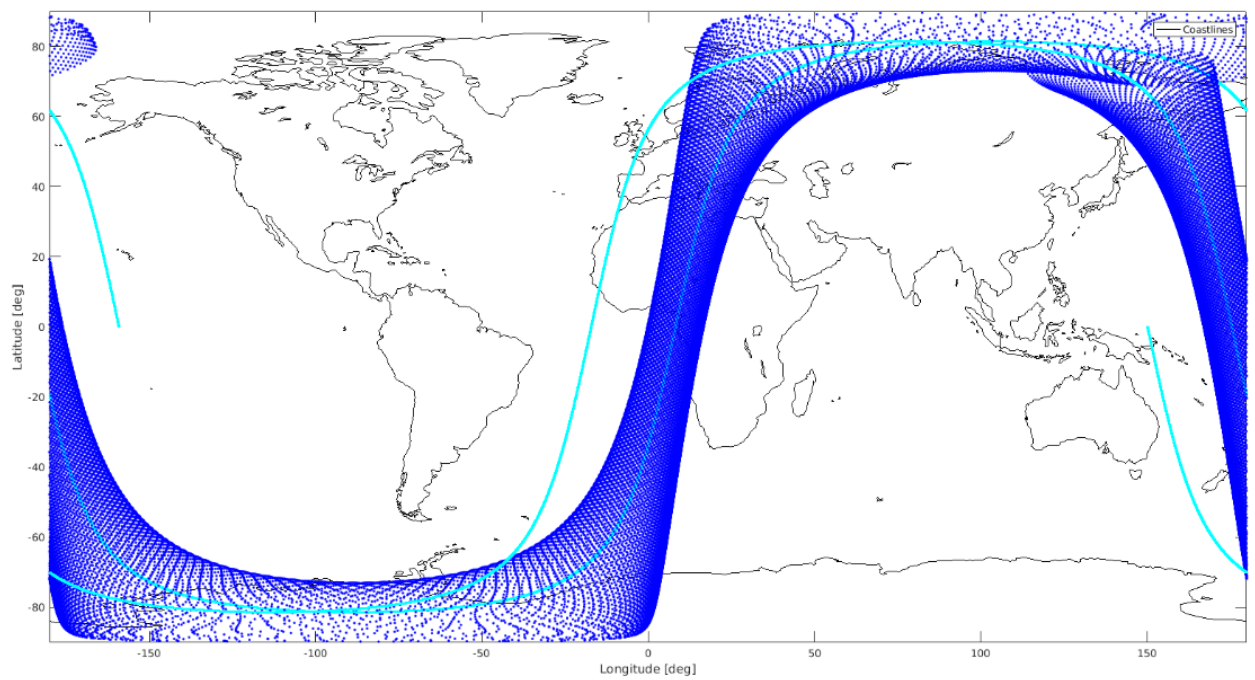

Figure 6. Full orbit of conical scanning microwave radiometer. 


\subsection{Passive Microwaves Instrument}

BIBLOS components currently support Passive Microwave Instruments working in conical scanning mode, for instance, Microwave Imager (MWI) [9]. Attitude parameters, such as rotation speed, integration time or look angle, can be controlled using configuration files. Intersection with the ground (Figure 7) is handled by EOCFI library [6], example result is shown in Figure 8. Scene is generated with user-defined maps of parameters, one of which is terrain type, as seen in Figure 9. Brightness temperature of the terrain is propagated through the atmosphere. Instrument processing is controlled by configuration files, where noises, calibrations and various parameters of system response and antenna pattern can be defined. The output is later processed by L1PM, which is reconstructing brightness temperatures on top of atmosphere, as presented in Figure 10.

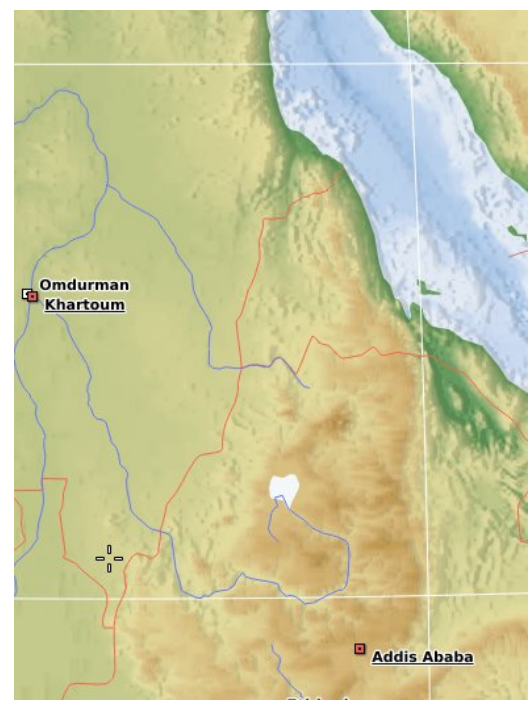

Figure 7. Map of observed terrain in example scenario.

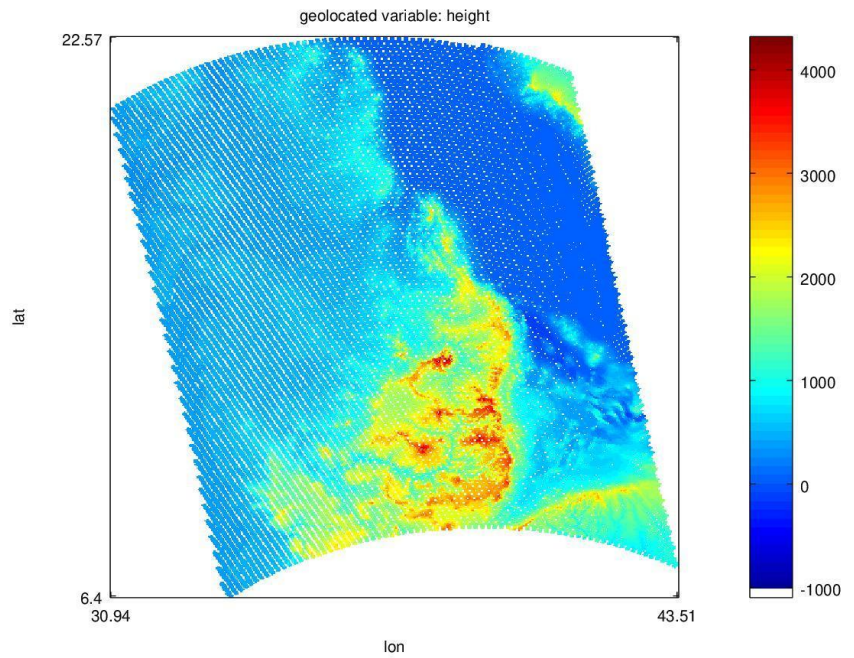

Figure 8. Terrain elevation map [m]. 


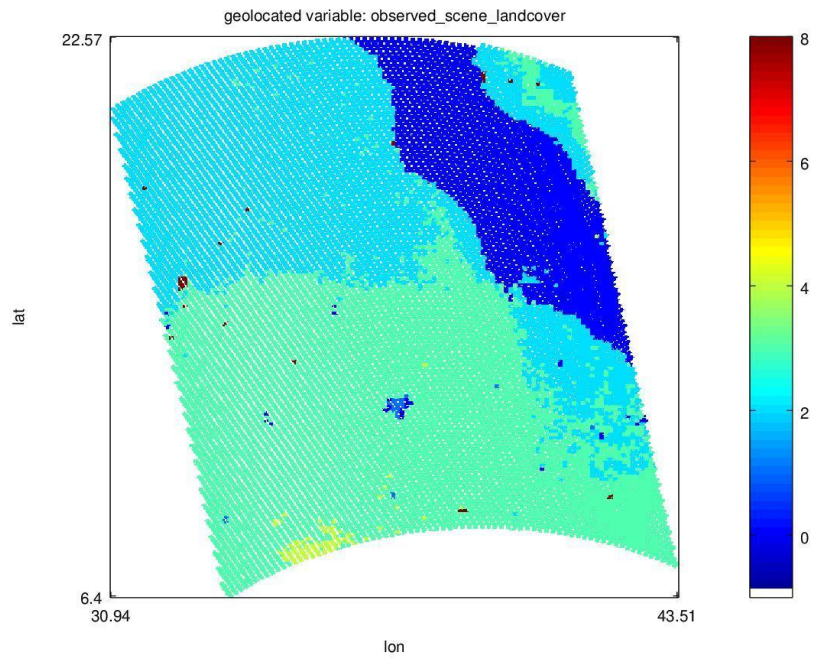

Figure 9. Terrain type, presented as integer number

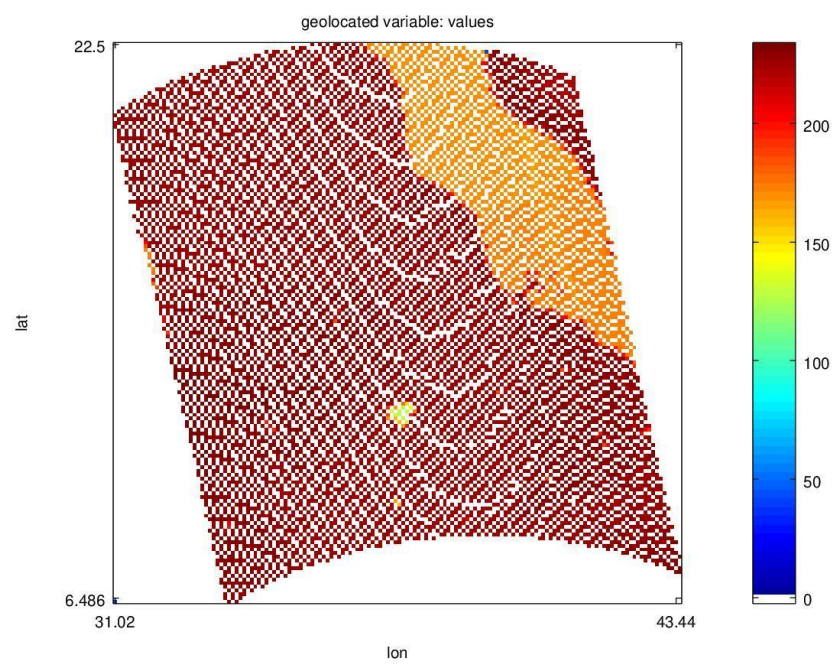

Figure 10. Reconstructed brightness temperature [K] on top of atmosphere - output of L1PM.

\subsection{Active Microwaves Instrument}

Based on the modified architecture [1] a multistatic mission configuration with three satellites has been investigated to prove BIBLOS usage for performance analysis of active microwave instrument. Defined scenario assumes one active transmitter satellite $(\mathrm{T} x 1)$ and two receiver satellite $(\mathrm{R} \times 1, \mathrm{R} \times 2)$. Configuration $\mathrm{Tx} 1-\mathrm{T} \times 1$ represents monostatic configuration [10], whereas Tx1-Rx1 and Tx1-Rx2 are two configurations with passive companion satellites (CS). Table 1 shows orbit initialization and beam steering parameters of considered scenario. Following CSs (Rx1, Rx2) are initialized with various orbit state vectors at ascending node crossing time. Orbit and attitude files are calculated by the GM (compliant with 6. Example figures presented below were automatically generated by PEM which has been fed with data generated by blocks of the GM and SGM (see Figure 1 for data flow between modules). Figure 11 shows the diamond diagram, a crucial parameter for assessment of observation geometry vs. instrument setting and pointing. Five horizontal lines represent look angles for five SAR beams each working with different PRF. Black and red lines represent, respectively, transmission period and nadir returns. Scene has been modelled based on complex constitutive parameters to include losses. 
Figure 12, Figure 13 and Figure 14 show the two-way antenna patterns for considered bistatic configurations, respectively, Tx1-Tx-1, Tx1-Rx1 and Tx1-Rx2. Within the BIBLOS-2 project the PEM module is designed to evaluate performance figures, such as diamond diagram, baselines, bursts and swath location, out-of-plane angle, location of the reception windows, one-way and two-way antenna pattern, slant range resolution, signal-tonoise ratio, noise equivalent sigma zero, radiometric resolution, azimuth resolution, range, azimuth and total ambiguity ratios for a theoretical point target and distributed target, theoretical instrument response functions (IRF), for chosen multistatic mission configuration.

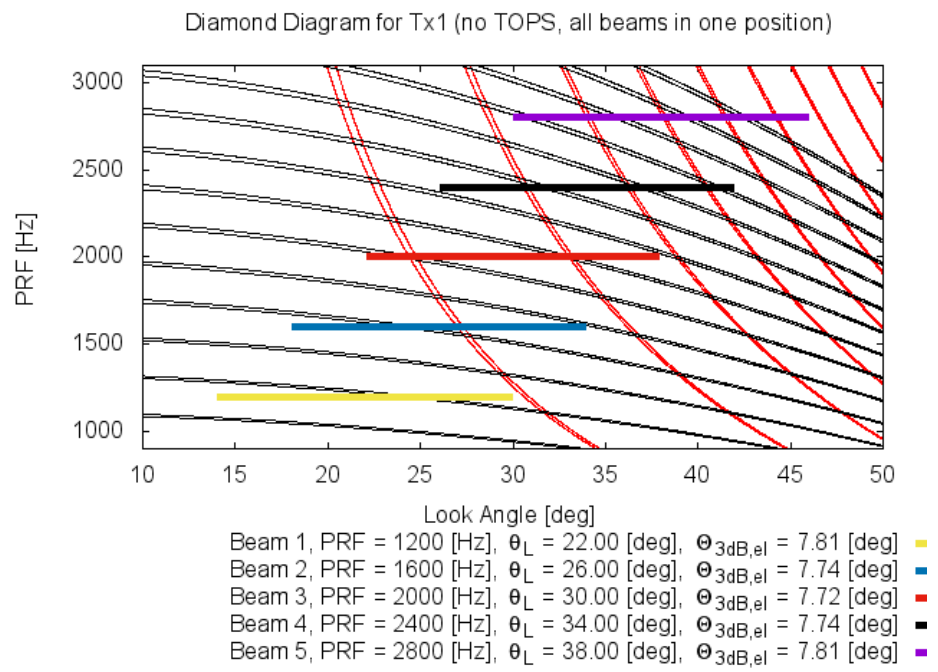

Figure 11. Diamond Diagram for Tx1-Tx1.

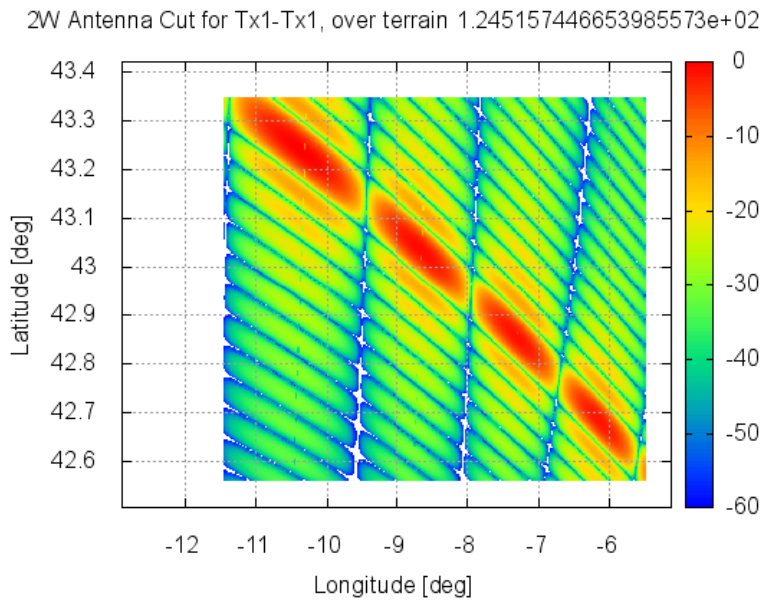

Figure 12. Two-way antenna pattern for Tx1-Tx1.

\section{CONCLUSION AND FUTURE PLANS}

BIBLOS-2 project was successfully ended and in mid of October 2019 entered into the Maintenance Period. Software library for creating E2ES EO was created as planned. Current phase of the project extended the EOMODEL (BIBLOS-1) library by adding ready-to-use software Building Blocks for Active and Passive Microwave 


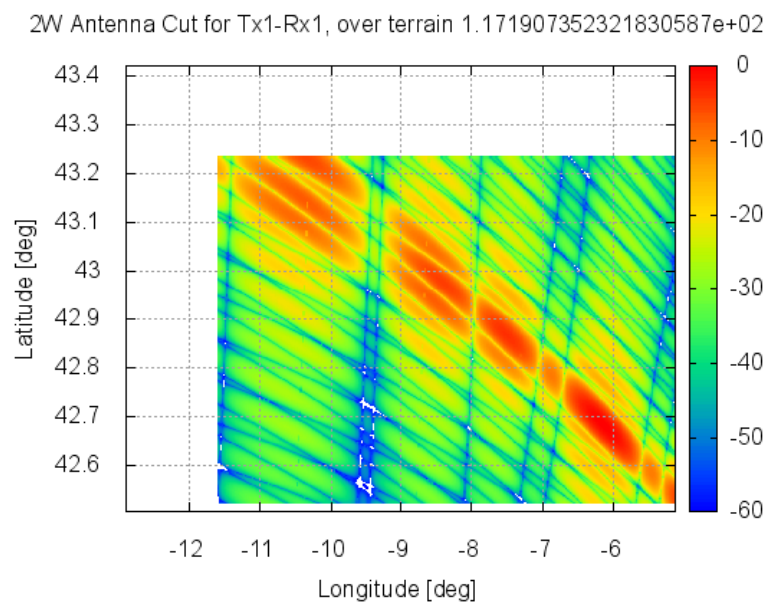

Figure 13. Two-way antenna pattern for Tx1-Rx1

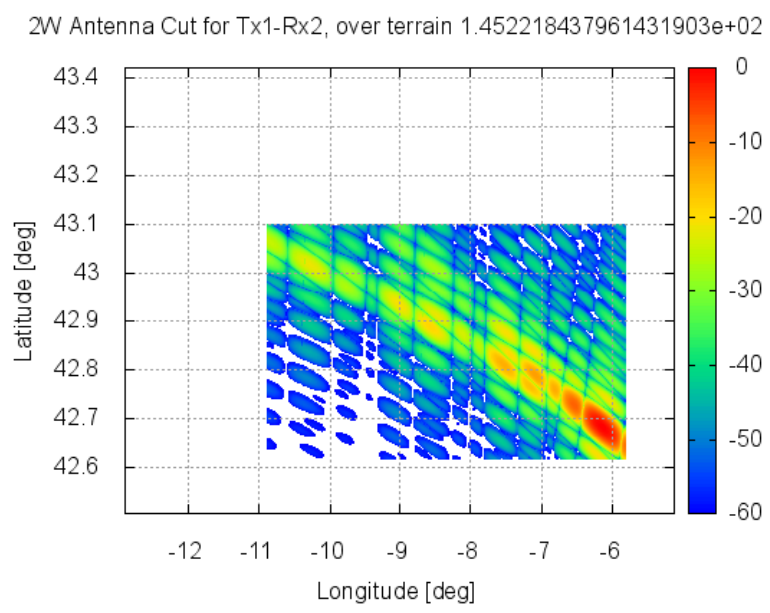

Figure 14. Two-way antenna pattern for Tx1-Rx2.

instruments. Updated website provides an access to software components as well as to dedicated installation packages (GNU / Linux and MacOs) and step-by-step guides to facilitate installation procedure for not softwareoriented users. Together with a variety of tutorials, planned to be released by end of 2019, available content will serve for EO community. More detailed documentation can be used by advanced users. Lessons learned during the second phase significantly contributed to a more precise definition of the scope for the next BIBLOS phase, referred to as BIBLOS-3. During the second phase the Building Blocks implemented for passive microwave instrument have been re-used and tailored for Instrument Data Simulator and Ground Prototype Processor of Operational Simulator for a conical scanning microwave radiometer developed within the Copernicus Imaging Microwave Radiometer (CIMR) mission. This indicates benefits of the BBs and proves the concept of cost reduction and fast prototyping, especially in the early stages of the project. An additional benefit is that standardized interfaces of BBs allow comparison of results for changed algorithms between various versions of E2ES as well as between BBs tailored for operational simulator.

Several tasks are planned for maintenance period of BIBLOS-2 project. Mostly, Adaptive and Corrective 
Maintenance will be perform to take advantage of the last version of EOCFI, OSFI, OpenSF and others. Several corrections are planned in already developed Building Blocks, mostly, to take into account additional parameters or modify equations. In general, all Building Blocks will be reviewed and updated to benefit from new EOCFI functionalities as well as to provide compatibility with new API. In the active microwave instrument the external interfaces of the Performance Blocks will be modified to provide an automatic filename creation and figure generation without entering filenames as input parameters. Moreover, additional flag will be added to configuration file to define swath width by ground distance, not only by elevation beamwidth. Upgrade is also planned in AOCS Blocks and BBs comprising the Scene Generation Module. In passive microwave instrument the BBs comprising the Level-1 Processing Module will be updated with new calibration algorithms. In addition, Preventive Maintenance is planned to be performed as modification of Building Blocks resulting from the Software Problem Reports (SPRs) reported by users. It is expected that BIBLOS component will be widely used in EO Community and this will directly translate into a number of comments and suggestions for improvement. Any suggestion, comments and remarks can be sent directly to BIBLOS Team at biblos@gmv.com. Moreover, additional efforts is planned and agreed with ESA to popularize the use of BIBLOS components both by participating in conferences and seminaries and by preparation of training and working sessions. Direct meetings with users will be used to gather feedback. In the next phase, i.e. BIBLOS-3, the same of software components are planned to be parallelized, for instance, AOCS Blocks, and installation procedure is being considered for virtualization. Moreover, new BBs are proposed to be designed to support Imaging Spectrometers for passive optical instrument. In active microwave, design, implementation and verification of Instrument and Level-1 Processing Modules is planned to make it possible to create entire E2ES. In passive microwave, the Performance Evaluation Module is planned to be added.

\section{ACKNOWLEDGMENTS}

BIBLOS-2 Project is led by GMV Innovating Solutions Sp. z o.o. and realized for the European Space Agency under the Contract No. 4000118711/16/NL/AF "Model Library for Earth Observation End-to-End Simulators".

\section{REFERENCES}

[1] BIBLOS Website. https://gmv-biblos.gmv.com/. Accessed: 2019-12-18.

[2] BIBLOS Team, “BIBLOS-1 Final Report (GMV-BIBLOS-FR)," (2016).

[3] ARCHEO-E2ES Team, "ARCHEO-E2E FINAL REPORT (GMV-ARCHEO-E2E-FR-001)," (2013).

[4] ESA, GMV, “EO E2E2 Reference Architecture (ARCHEO-E2E-TN-002), v3.1,” (2013).

[5] ESA, GMV, “EO E2E2 Reference Architecture (ARCHEO-E2E-TN-002), v3.2,” (2018).

[6] Earth Observation CFI Software. http://eop-cfi.esa.int/index.php/mission-cfi-software/ eocfi-software. Accessed: 2019-10-15.

[7] "European Space Agency - ESA Software Community Licence Permissive."

[8] EOP-PE CFI Team, "Earth Observation Mission Software File Format Specification, PE-ID-ESAGS-584, version 1.4." http://eop-cfi.esa.int/REPO/PUBLIC/DOCUMENTATION/SYSTEM_SUPPORT_DOCS/ obsolete/PE-ID-ESA-GS-584-1.2-EO_Mission_SW_File_Format_Specs.pdf.

[9] The Microwave Imager (MWI). https://www.eumetsat.int/website/home/Satellites/ FutureSatellites/EUMETSATPolarSystemSecondGeneration/MWI/index.html. Accessed: 2019-1015.

[10] Sentinel-1 (SAR). https://www.eumetsat.int/website/home/Satellites/FutureSatellites/ EUMETSATPolarSystemSecondGeneration/MWI/index.html. Accessed: 2019-10-15. 